\title{
Phylogeography of the planktonic shrimp Lucifer hanseni Nobili 1905 in the Indo- Malayan Archipelago
}

\begin{abstract}
Using partial sequences of two mitochondrial genes, cytochrome c oxidase subunit I (COI) and $12 \mathrm{~S}$ ribosomal RNA (12S rRNA), and one nuclear gene, 28S ribosomal RNA (28S rRNA), we investigated population genetics of the holoplanktonic shrimp Lucifer hanseni Nobili, 1905 in the Indo-Malayan Archipelago (IMA), encompassing Andaman Sea, Malacca Strait, Gulf of Thailand, Borneo Island, Philippines (hereafter collectively referred to as the Thailand-Malaysia-Philippine area: TMP), Celebes Sea (CS), and the waters near islands in the Western Pacific (WP) including Palau, Papua New Guinea and Solomon Islands. The samples from the TMP showed the highest number of haplotypes. Significant phylogeographic structure was found in the L. hanseni populations ( $\overline{\mathrm{ST}}=0.832$ for COI, 0.159 for $12 \mathrm{~S}$ rRNA, 0.783 for $28 \mathrm{~S}$ rRNA). The total number of haplotypes was 46 in COI, 28 in $12 \mathrm{~S}$ rRNA and 23 in 28S rRNA. The haplotype network analyses revealed two major clades for COI (subgroups: TMP + CS, WP) and for 12S rRNA and 28S rRNA (TMP, CS + WP). The CS and WP populations appeared isolated from the TMP populations. The samples from the CS showed low genetic diversity compared with the other samples at both haplotype and nucleotide levels, suggesting that the population CS experienced bottleneck events. This is the first demonstration of significant genetic structure of a holoplanktonic metazoan in IMA, which is suggested to be synergistically influenced by historical events (vicariance) and contemporary oceanographic circulations and corroborates the results of previous studies on other benthic/demersal animals with mero-planktonic phases.
\end{abstract}

Keyword: Indo-Malayan Archipelago; Lucifer hanseni; Phylogeography 